\title{
Lethal Factor Domain-Mediated Delivery of Nurr1 Transcription Factor Enhances Tyrosine Hydroxylase Activity and Protects from Neurotoxin-Induced Degeneration of Dopaminergic Cells
}

\author{
Dennis Paliga $^{1} \cdot$ Fabian Raudzus ${ }^{1} \cdot$ Stephen H. Leppla ${ }^{2} \cdot$ Rolf Heumann $^{1}$ (D) $\cdot$ Sebastian Neumann ${ }^{1}$
}

Received: 16 February 2018 / Accepted: 8 August 2018 / Published online: 18 August 2018

(C) The Author(s) 2018

\begin{abstract}
The orphan transcription factor nuclear receptor-related 1 protein (Nurr1, also known as NR4A2) plays a key role in embryonic development and maintenance of mesencephalic dopaminergic neurons in the substantia nigra. Nurrl deficiency is associated with Parkinson's disease where dopaminergic neurons degenerate suggesting that counter-regulation of Nurrl activity may have therapeutic effects. Here, we bacterially expressed and isolated a human Nurrl fusion protein containing a N-terminal cell delivery domain derived from detoxified anthrax lethal factor followed by wild type ubiquitin with deubiquitinating enzyme recognition site for intracellular cleavage. Addition of the Nurrl fusion protein to dopaminergic SH-SY5Y cells generated a cleaved, cytosolic Nurrl-containing fragment which was associated with increased levels of tyrosine hydroxylase, the ratelimiting enzyme in dopamine synthesis. Promoter-activity assays confirmed that exposure of cells to full-length Nurrl fusion protein activated not only its cognate human tyrosine hydroxylase promoter but also the corresponding mouse sequence, although at a reduced efficiency. Using 6-hydroxydopamine as a dopaminergic cell specific neurotoxin, we demonstrate that full-length Nurrl fusion protein promotes a concentration-dependent protection from this toxic insult. Altogether, the enhancement of tyrosine hydroxylase in naïve dopaminergic cells and the protective effects in a cellular model of Parkinson's disease suggest that full-length Nurrl fusion protein may contribute to the development of a novel concept of protein-based therapy.
\end{abstract}

Keywords Nurr1 $\cdot$ SH-SY5Y $\cdot$ Lethal factor $\cdot$ Cellular delivery $\cdot$ Fusion protein $\cdot$ Cellular protection

\section{Introduction}

Nuclear receptor-related 1 protein (Nurr1, also known as NR4A2) is a member of the NR4A superfamily of nuclear receptor proteins including Nur77 (NR4A1) and Nor1 (NR4A3). Nurr1 has been shown to be involved in the

Rolf Heumann and Sebastian Neumann contributed equally to this work and share last authorship.

Electronic supplementary material The online version of this article (https://doi.org/10.1007/s12035-018-1311-6) contains supplementary material, which is available to authorized users.

Rolf Heumann

rolf.heumann@ruhr-uni-bochum.de

1 Department of Biochemistry II - Molecular Neurobiochemistry, Faculty of Chemistry and Biochemistry, Ruhr-Universität Bochum, 44801 Bochum, Germany

2 Laboratory of Parasitic Diseases, National Institute of Allergy and Infectious Diseases, National Institutes of Health, Bethesda, MD, USA development and maintenance of adult dopaminergic neurons in the midbrain. However, Nur77 and Nor1 have other functions, such as the induction of apoptotic pathways [1-4].

Nurr1 is an orphan transcription factor that influences the expression of several key proteins of dopaminergic (DA) neurons, including tyrosine hydroxylase $(\mathrm{TH})$, dopamine transporter (DAT), and vesicular monoamine transporter (VMAT) [5]. Furthermore, Nurr1 is involved in the regulation of a complex network of pathways such as Pitx 3 and $\mathrm{Wnt} / \beta$-catenin, thereby influencing the neurogenesis of dopaminergic cells $[6,7]$. Altogether, the development, differentiation, and survival of DA neurons depend on Nurr1 [5]. Although the vast majority of Parkinson's disease (PD) cases are sporadic, about $10 \%$ of the cases are based on genetic factors including mutations in about 18 genes [8]. The morbidity of DA neurons of elderly PD patients might be explained by an agedependent decline in Nurr1 expression [9]. Consistently, overexpression of Nurrl mediates anti-inflammatory effects and neuroprotection in PD models - in vitro and in vivo [10] and recent reviews emphasize the potential of using Nurr1 in PD therapy [11]. Nurr1-based gene therapy has been 
successful in animals after injection of adeno-associated virus encoding Nurr1/Foxa2, but unfortunately, critical questions remain to be answered before viral vector-based delivery can be applied to patients [12]. Application of drugs that activate Nurr1 or enhance its expression level have been promising, but questions of drug specificity of action have been put forward $[11,13]$. Here, we aim to contribute to the discussion of a protein-based method of Nurr1 application with the option of a reversible and tunable delivery of Nurr1.

In order to allow efficient nuclear delivery of extracellular applied transcription factor Nurr1, we made use of the mechanism by which the Gram-positive bacterium Bacillus anthracis causes the anthrax disease; its virulence is mediated by the poly- $\gamma$-D-glutamic acid capsule and by the secreted anthrax toxin (AT). The three proteins protective antigen (PA, $83 \mathrm{kDa})$, lethal factor (LF, $90 \mathrm{kDa})$, and edema factor (EF, $89 \mathrm{kDa}$ ) build up anthrax toxin (AT). Whereas each of these three proteins is not individually toxic, lethality was shown for the combinations of LF together with PA and EF along with PA [14]. PA is required for enabling LF and EF to enter the host cells. PA binds to one of the ubiquitously expressed cell surface receptors tumor endothelial marker 8 (TEM8 or ANTRXR1) or capillary morphogenesis gene 2 (CMG2 or ANTRX2) [15]. Upon binding, PA becomes cleaved by furin protease resulting in a $63 \mathrm{kDa}$ protein that oligomerizes into a ring-shaped heptamer or octamer forming a channel which binds LF and EF. This complex is taken up by clathrin-dependent endocytosis into endosomes [16]. The intra-endosomal $\mathrm{pH}$ decrease leads to the insertion of the PA oligomer into the endosomal membrane forming a pore [17]. Unfolded LF and EF can translocate through this pore driven by the $\mathrm{pH}$ gradient and are released into the cytosol [18]. Finally, chaperones are needed for the refolding of LF and EF [19].

In 1992, Arora et al. fused full-length LF with the ADPribosylation domain of Pseudomonas exotoxin A as a cargo and demonstrated its PA-dependent cellular uptake into mammalian cells [20]. The non-toxic N-terminal amino acids 1254 of LF (LFn) are sufficient for the cellular delivery of fused proteins along with PA [21]. However, some studies report delivery of LFn fusion proteins independent from PA and more recently, it has been shown that PA-dependent and PAindependent delivery of peptides may co-exist because stimulation of $\mathrm{CD}^{+}$T-cells of the immune system by LFn fusion proteins does not require, but is enhanced by, PA in vitro [22-25].

In addition to Nurr1 and its cell delivery domain LFn, we used ubiquitin and small ubiquitin-like modifier (SUMO) protein. SUMO can be attached to target proteins as posttranslational modification for diverse cellular processes [26]. Furthermore, fusion proteins with SUMO are useful for the heterologous protein expression because it may increase the amount of the recombinant expressed protein and may increase its solubility and enhance its stability [27]. To ensure nuclear translocation of transcriptionally-active Nurr1 after LFn-mediated delivery into the cytosol, we wanted to achieve its proteolytic cleavage from the fusion protein using suitable cytosolic proteases. Deubiquitinating enzymes (DUBs) are cytosolic or endosome-associated proteases that counteract ubiquitination by recognizing the di-glycine motif at the C-terminus of ubiquitin and releasing ubiquitin from proteins or from ubiquitin fusion proteins [28].

In this study, we investigate the application of the non-toxic $\mathrm{N}$-terminal part of lethal factor from $B$. anthracis for cellular delivery of Nurr1 as a fusion protein with SUMO and ubiquitin. Following bacterial expression of this fusion protein, we use a tyrosine hydroxylase promoter assay for quantifying the biological activity of Nurr1 after cellular uptake into the human neuroblastoma line SH-SY5Y. Furthermore, we examine in the presence or absence of PA the possible protective effects of this Nurr1 fusion protein after treatment of human $\mathrm{SH}$ SY5Y cells with the neurotoxin 6-hydroxydopamine (6OHDA).

\section{Materials and Methods}

\section{Construction and Purification of Nurr1 Fusion Proteins and PA}

The HS-LUNN1 fusion protein was constructed by combining the LFn open reading frame (ORF) from pET-15b-LFN WT (a gift from John Collier, Addgene plasmid \#11082, Addgene, Teddington, UK), the ubiquitin ORF from pET15-ubiquitin WT [29] (a gift from Rachel Klevit, Addgene plasmid \#12647, Addgene), and the Nurr1 sequence from pMX-HTNN into the Champion ${ }^{\mathrm{TM}}$ pET SUMO Expression System (Fisher Scientific GmbH, Schwerte, Germany) via a combination of traditional and infusion cloning (CLONTECH, Takara Bio Europe SAS, Saint-Germain-enLaye, France). The main features of the Champion ${ }^{\mathrm{TM}}$ vector system include an inducible $\mathrm{T} 7$ promoter, an $\mathrm{N}$-terminal hexahistidine tag for protein purification, multiple cloning sites, and an internal SUMO sequence, which is believed to increase solubility of hard to express proteins and can be cleaved by SUMO protease to get untagged proteins. The human Nurr1 coding sequence (accession number NM_006186.2) in pMX-HTNN was codon optimized with GeneOptimizer® (Thermo Fisher Scientific, MA, USA) for bacterial expression and was synthesized by GeneArt ${ }^{\circledR}$ (Thermo Fisher Scientific). The fusion protein variants $\mathrm{H}$ LUNN1, HS-NN1, and H-N1 resulted from sequential deletion mutation with Q5® Site-Directed Mutagenesis Kit (New England BioLabs, Frankfurt am Main, Germany) of the fulllength construct pET-HS-LUNN1. All constructs were 
confirmed by DNA sequencing. Detailed primer sequences are listed in Supplements (Supplement Tables 1 and 2). For protein expression, the constructs were transformed into the Escherichia coli (E. coli) strain Rosetta-gami ${ }^{\mathrm{TM}} 2$ (DE3) pLysS (Merck Millipore, Darmstadt, Germany), grown in terrific broth (TB) media at $37{ }^{\circ} \mathrm{C}$ until an optical density of $\mathrm{OD}_{600}=0.9-1.1$ and expression was induced with $1 \mathrm{mM}$ isopropyl $\beta$-D-1-thiogalactopyranoside (IPTG, Fisher Scientific $\mathrm{GmbH})$. According to manufacturer's protocol, cells were lysed and the fusion proteins were purified from bacterial cell extracts by metal chelation using nickel nitrilotriacetic acid (Ni-NTA) beads (Qiagen, Hilden, Germany). The purified Nurr1 fusion proteins have a predicted molecular mass of $\approx 118 \mathrm{kDa}$ for HS-LUNN1, $\approx 107 \mathrm{kDa}$ for H-LUNN1, $\approx 81 \mathrm{kDa}$ for HS-NN1, and $\approx 67 \mathrm{kDa}$ for H-NN1. The production and purification of PA were similarly accomplished by using pET-22b-PA WT (a gift from John Collier, Addgene Plasmid \#11079, Addgene) in E. coli and metal chelation purification via $\mathrm{C}$-terminal hexahistidine tag resulting in the $\approx 83$ $\mathrm{kDa}$ sized protein. All proteins were dialyzed in Dulbecco's modified Eagle's medium (DMEM, Sigma-Aldrich, Steinheim, Germany) Amicon centrifugation tubes (Merck Millipore) with a molecular weight cut-off of 30,50, or $100 \mathrm{kDa}$ according to protein size following manufacture's protocol.

\section{Cell Culture}

The human neuroblastoma cell line SH-SY5Y (CRL-2266) was obtained from the American Type Culture Collection (ATCC, Rockville, USA) and routinely grown at $37{ }^{\circ} \mathrm{C}$ in a 1:1 ratio of DMEM (Sigma-Aldrich) and Ham's F-12 (Sigma-Aldrich) supplemented with $2 \mathrm{mM}$ glutamine (Sigma-Aldrich), 10\% ( $v / v)$ fetal calf serum (MerckBiochrom, Berlin, Germany), and $25 \mu \mathrm{g} / \mathrm{ml}$ penicillin/streptomycin (Gibco, Fisher Scientific $\mathrm{GmbH}$ ) in a humidified atmosphere of $5 \% \mathrm{CO}_{2}$ in the air. Identical cell numbers were seeded in 96-well, 24-well, or 6-well plates and grown to 70$80 \%$ confluence depending on experimental needs. Transfection was performed using ViaFect ${ }^{\mathrm{TM}}$ transfection reagent (Promega, Mannheim, Germany). To verify transfection efficiency, pcDNA3-eGFP (a gift from Doug Golenbock, Addgene plasmid \#13031, Addgene) was cotransfected, and eGFP fluorescence was observed via fluorescence microscopy (not shown).

\section{Luciferase Reporter Assay}

Cells were co-transfected with firefly luciferase encoding reporter plasmids pGL3-B (promoterless vector, E1751, Promega), pTHm-pGL3-B (mouse TH promoter), pTHhpGL3-B (human TH promoter), and the internal control plasmid pRL-TK (E2241, Promega,) expressing Renilla luciferase (if not otherwise stated). Cells were incubated for $24 \mathrm{~h}$ with transfection reagent, washed with phosphate buffer saline (PBS), and subsequently treated with different concentrations of fusion protein $(0-10 \mu \mathrm{M})$ for additional $24 \mathrm{~h}$. After several washing steps, cells were lysed and luciferase signals were measured following manufacturer's protocol (Dual-Glo® luciferase assay system, Promega) with 1420 luminescence counter Victor ${ }^{\circledR}$ light (PerkinElmer LAS, Rodgau, Germany).

\section{Cell Viability Assay}

Neurotoxicity assays with 6-hydroxydopamine (SigmaAldrich) were carried out by quantifying luciferase signals produced from substrate conversion of living cells with RealTime-Glo ${ }^{\text {TM }}$ MT cell viability assay (Promega) to determine cell viability after fusion protein and toxin treatment. Cells were plated in equal cell numbers; and after incubation with different concentrations of HS-LUNN $1 \pm \mathrm{PA}(0-3 \mu \mathrm{M}$, proteins in equimolar concentrations) for $24 \mathrm{~h}$, cells were washed three times with PBS and incubated with different concentrations of 6-OHDA $(0-200 \mu \mathrm{M})$ for $1 \mathrm{~h}$ at $37^{\circ} \mathrm{C}$ followed by the viability determination procedure mentioned before. Signals were measured following manufacturer's protocol with microplate reader CLARIOstar® (BMG Labtech, Ortenberg, Germany).

\section{Cell Counting}

Alternatively, intoxicated cells were counted according to the following protocol: 10,000 cells were seeded per well in a 96 well plate. The next day, different concentrations of HSLUNN1 $\pm \mathrm{PA}(0-3 \mu \mathrm{M}$, proteins in equimolar concentrations) were applied in quadruplicates including controls without any protein. After $24 \mathrm{~h}$, the cells were washed three times with PBS, and different concentration of 6-OHDA were applied for $1 \mathrm{~h}$ at $37^{\circ} \mathrm{C}$. After $24 \mathrm{~h}$, the cells were fixed in PBS supplemented with $4 \%$ PFA and $0.5 \%$ glutaraldehyde for $15 \mathrm{~min}$ and permeabilized with $0.5 \%$ Triton-X 100 in PBS for $15 \mathrm{~min}$ at room temperature. Staining was performed with $0.1 \mu \mathrm{g} / \mathrm{ml}$ 4',6-diamidino-2-phenylindole (DAPI) in PBS for $10 \mathrm{~min}$ at room temperature. Finally, the cells were washed with PBS for 5 min three times and kept in PBS. Micrographs were taken using a wide field fluorescence microscope (Olympus IX51, Hamburg, Germany) at $\times 10$ magnification. These images were used for cell counting with the help of the software ImageJ [30].

\section{Western Blot Analysis}

Recombinant proteins and whole-cell lysates were subjected to SDS-PAGE (8-10\%, Tris- $\mathrm{HCl})$ and then blotted onto nitrocellulose membrane (GE Healthcare Life Sciences, 
Freiburg, Germany). SH-SY5Y cells prepared for whole-cell protein immunoblot detection were treated with $0.25 \%$ Trypsin-EDTA (Sigma-Aldrich) PBS to detach cells and remove remaining protein before lysis. To preserve proteins from degradation, we used the lysis protocol and the passive lysis buffer included in the Dual-Glo® luciferase assay system (Promega) mentioned before. After determination of protein concentration, samples were mixed with Laemmli buffer and heated for at least $5 \mathrm{~min}$ at $95{ }^{\circ} \mathrm{C}$. Membranes were blocked with $5 \%$ non-fat dry milk in PBS, then incubated for $2 \mathrm{~h}$ at room temperature or overnight at $4{ }^{\circ} \mathrm{C}$ with primary antibody solution. Detailed antibody information is listed in Supplements. The membranes were then washed repeatedly and incubated with horseradish peroxidaseconjugated (HRP) secondary antibody solution for $1 \mathrm{~h}$ or with alkaline phosphatase-conjugated (AP) secondary antibody solution for $2 \mathrm{~h}$ at room temperature. Reactions were developed using SuperSignal ${ }^{\mathrm{TM}}$ West Pico chemiluminescent substrate (Fisher Scientific GmbH) for HRP secondary antibodies and visualized on X-ray film (GE Healthcare Life Sciences, Freiburg, Germany) or with ChemiDoc ${ }^{\mathrm{TM}}$ XRS+ system (BIO-RAD) and with $\mathrm{BCIP} \circledast \mathrm{NBT}$ liquid substrate (Sigma-Aldrich) for AP secondary antibodies for direct staining of the membrane.

\section{Protein Concentration Estimation}

Protein concentrations were determined by using DC ${ }^{\text {TM }}$ protein assay (BIO-RAD) and colorimetric measurement performed at $670 \mathrm{~nm}$ with absorbance microplate reader Sunrise ${ }^{\mathrm{TM}}$ (Tecan Deutschland GmbH, Crailsheim, Germany).

\section{Scanning and Analysis of the Images}

De-stained gels, X-ray films, and dried blots were scanned using the SHARP MX-4141 N PS (Sharp electronics business systems, Cologne, Germany) unless pictures were taken directly via the integrated camera of ChemiDoc ${ }^{\mathrm{TM}} \mathrm{XRS}+$ system (BIO-RAD) mentioned before. All scans were performed in professional mode at $600 \mathrm{dpi}$ and in 16-bit grayscale. All figures were created with the software Microsoft PowerPoint 2016 (Redmond, Washington, USA).

\section{Statistics}

Protein bands were analyzed using ImageJ (NIH, Bethesda, MD, USA) software. The dose response curve was created using the software Prism version 7.04 for Windows (GraphRad, San Diego, CA, USA). Data are shown as mean \pm standard error of mean (SEM). Statistical significance was determined using the $t$ test analysis normalized to untreated cells. Experiments were replicated at least three times; $p$ values under 0.05 were considered significant.

\section{Results}

\section{Fragments of Full-Length Nurr1 Fusion Protein Are Delivered into SH-SY5Y Cells}

Previously, Bachran et al. [31] established a fusion protein consisting of LFn, followed by ubiquitin and fused to protein of interest, the Pseudomonas exotoxin A catalytic domain (PEIII). Here, we added N-terminal SUMO to this system and introduced a nuclear localization signal (NLS) to the Nurr1 protein (Fig. 1a). We used SUMO in order to increase the solubility of the fusion protein during expression as described elsewhere [27]. Ubiquitin was fused with a GGGlinker to NLS-Nurr1 with the intention that after cellular uptake of HS-LUNN1, intracellular DUBs would cut HSLUNN1 into two fragments, one composed of SUMO, LFn, and ubiquitin, as well as a second fragment consisting of NLS-Nurr1, which would enter the nucleus. Altogether, the fusion protein HS-LUNN1 (predicted molecular weight, $118 \mathrm{kDa}$ ) consists of six functional domains as described in Fig. 1a.

After purification of bacterially expressed HS-LUNN1 (Fig. S1), the protein was added to cultured SH-SY5Y cells at various concentrations ranging between 0.1 and $3 \mu \mathrm{M}$ for 18-24 h. Because of the contrasting cellular effects of Nurr1 and Nur77 respectively, we used antibodies detecting both Nurr1 and Nur77 at the same time. We observed a HSLUNN1 concentration-dependent 9.1-fold increase (related to endogenous Nurr1, Figs. 1c, and $3 \mu \mathrm{M}$ HS-LUNN1) of the Nurr1-containing fragment (NN1, 67.4 kDa) (Fig. 1b, upper band in upper panel). Interestingly, the endogenous Nur77 protein levels (64 kDa, Fig. 1b, lower band) decreased inversely to added HS-LUNN1 concentrations (4.8-fold 3- $\mu \mathrm{M}$ HS-LUNN1). The identities of the bands for Nurr1 or Nur77, respectively, were confirmed by Western blots using specific antibodies (data not shown).

In order to analyze N-terminal DUB intracellular cleavage products complementary to the above described C-terminal Nurr1-containing fragment, we used antibodies against LF. We found a HS-LUNN1 concentration-dependent increase of the LFn-containing HS-LU-fragment (Fig. 1d, supplement Fig. S3a, right panel). Note that the predicted size of HS-LU is $52 \mathrm{kDa}$ but runs at a higher MW position in the gel which could be due to the previously described shift of the SUMO domain [32]. As a next step, we investigated well-described Nurrl downstream effectors and found that TH protein levels were increased by HS-LUNN1 again in a concentrationdependent manner (Fig. 1d) [5]. 


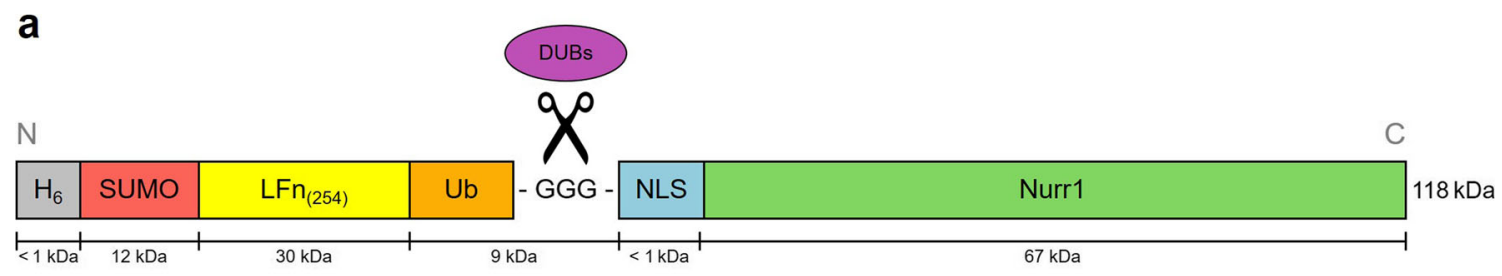

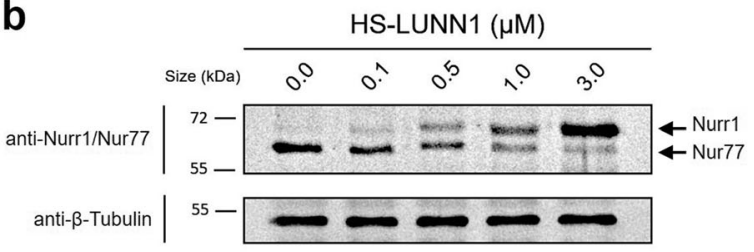

d

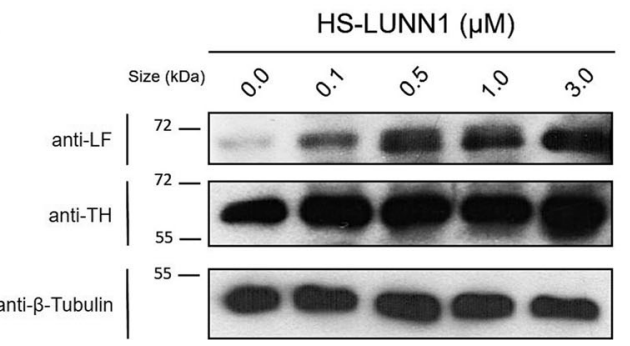

Fig. 1 Nurr1 protein delivery in SH-SH5Y cells. a Domain structure of Nurrl fusion protein consisting of hexahistidine purification tag $\left(\mathrm{H}_{6}\right)$, cleavable small ubiquitin-like modifier (SUMO), non-toxic N-terminal residue of Anthrax Lethal Factor (amino acid 1-254, $\operatorname{LFn}_{(254)}$ ), wild type ubiquitin with di-glycine motif GG'G (Ub) cleavable by deubiquitinating enzymes (DUBs), nuclear localization signal (NLS), and the human transcription factor Nurrl (HS-LUNN1). b, d Analysis of whole-cell lysates after treatment of SH-SY5Y cells with HS-LUNN1. All samples were taken $24 \mathrm{~h}$ after protein application and analyzed on a SDS-PAGE $(8 \%$ (b), $10 \%$ (d)) with primary antibodies anti-Nurr1/Nur77, anti-LF and anti-TH (d) by immunodetection following Western blotting.

\section{HS-LUNN1 Increases Levels of TH Protein and Activated Mouse and Human Tyrosine Hydroxylase Promoter Sequences}

In order to investigate possible downstream effects emerging from delivered Nurrl protein, we at first analyzed levels of TH protein in SH-SY5Y cells. There was a HS-LUNN1 concentration-dependent increase of TH protein detected by Western blotting (Fig. 1d). Next, we used a luciferase-based promoter assay [33] to directly test possible transcriptional activity by HS-LUNN1 after cellular delivery. The luciferase assay showed strong activation of mouse (pTHm) and human (pTHh) TH promoters (e.g., 1- $\mu$ M HS-LUNN1; pTHm 4.4 \pm 0.2 ; pTHh $14.5 \pm 0.8$ ). However, the human TH promoter is about threefold more strongly activated as compared to the mouse (Fig. 2). Moreover, saturation in luciferase activity was achieved already at concentrations at $1 \mu \mathrm{M}$ of HSLUNN1. Following the major difference in activation

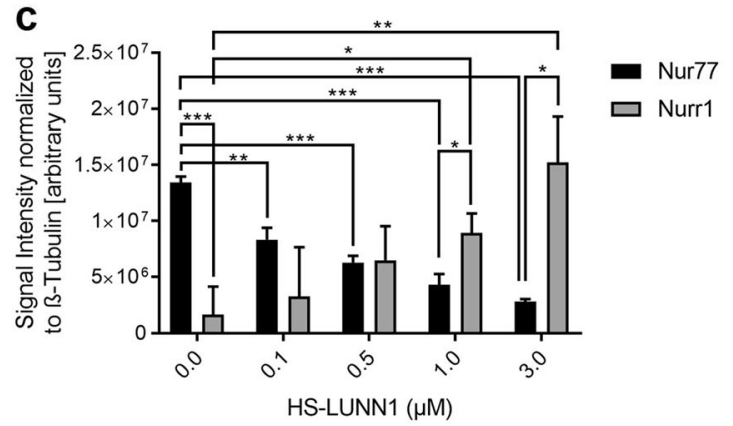

Each lane contained $2 \mu \mathrm{g}$ of whole-cell protein. The positions of molecular mass markers are shown to the left of the gels, and anti- $\beta$ Tubulin serves as loading control. Bands representative of three independent experiments are shown. $\mathbf{c}$ The band intensities of Nurr1 and Nur77 respectively were quantified and normalized to the band intensities of $\beta$-Tubulin. Bars represent mean \pm SEM obtained from three independent experiments and statistical significance was determined using $t$ test $(* p<0.05 ; * * p<0.01 ; * * * p<0.001 ; n=3, t$ test). Please note that endogenous Nurr1 (66.4 kDa) and Nurr1containing fragment $(\mathrm{NN} 1,67.4 \mathrm{kDa})$ have similar molecular weights

between the mouse and human $\mathrm{TH}$ promoter sequence by HS-LUNN1, we focused on the human TH promoter in all subsequent experiments. Performing a detailed dose response curve revealed that the lowest amount to activate the human $\mathrm{TH}$ promoter in the presence or absence of $\mathrm{PA}$ was in the range of $0.1-0.3 \mathrm{nM}$ of HS-LUNN1 (see Fig. S2).

\section{HS-LUNN1 Protects SH-SY5Y Cells from 6-OHDA Intoxication}

The neurotoxin 6-OHDA imposes stress to dopaminergic and noradrenergic cells by generating reactive oxygen species resulting in cellular degeneration [34]. To further investigate possible effects of HS-LUNN1 on 6-OHDA-induced stress, we first incubated SH-SY5Y cells with various concentrations of HS-LUNN1 for $24 \mathrm{~h}$ in the presence or absence of PA. Cells were then challenged with various concentrations of 6-OHDA $(50 \mu \mathrm{M}, 100 \mu \mathrm{M}$, and $200 \mu \mathrm{M})$ for $1 \mathrm{~h}$ at $37^{\circ} \mathrm{C}$ and washed 


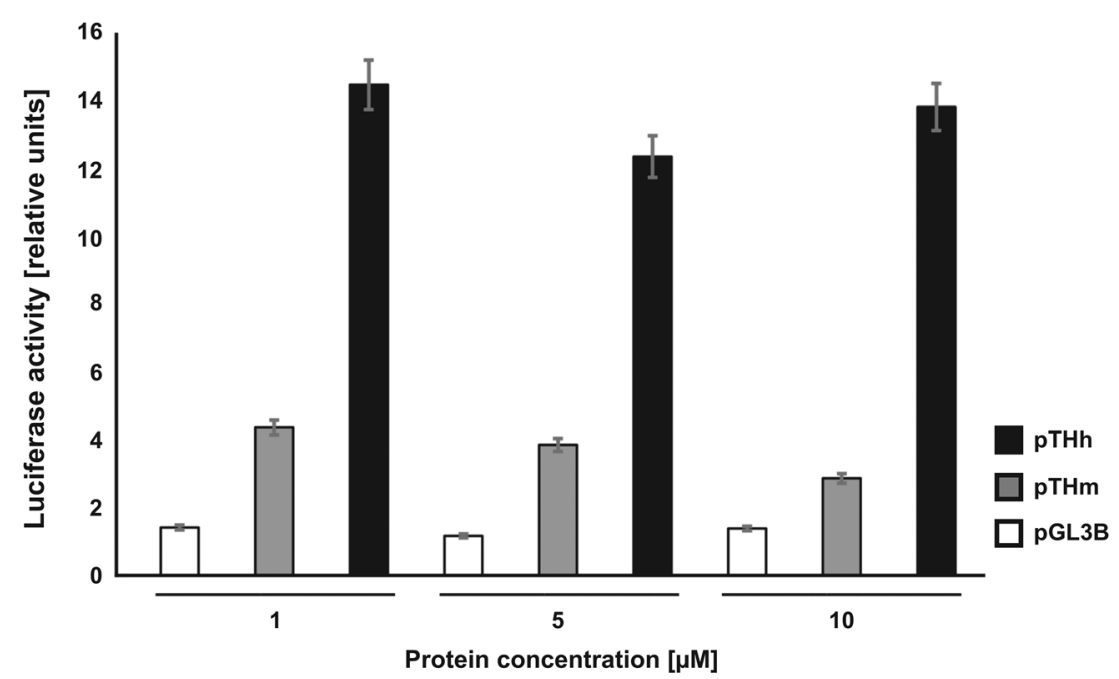

Fig. 2 Luciferase reporter assay in SH-SY5Y cells treated with HSLUNN1. Cells were transfected with pTHm-pGL3B (mouse TH promoter) and pTHh-pGl3B (human TH promoter) $24 \mathrm{~h}$ prior to protein incubation with HS-LUNN1. The Renilla luciferase expression plasmid, pRL$\mathrm{TK}$, and the original pGL3B (promoterless) control were utilized as an internal and external standard, respectively. Data are from two independent experiments, each of which was conducted in triplicate and expressed as means \pm standard error of the mean (SEM) for control cells (untreated cells only transfected with pTHh-pGl3B and pRL-TK). The experiment has been repeated twice with other concentrations with comparable results (data not shown)

truncated variants of full-length HS-LUNN1. H-LUNN1 (without SUMO domain) or HS-NN1 (without LFn and without ubiquitin) and H-N1 containing the Nurr1 protein without NLS (Fig. S3) were analyzed for their activity of activating TH promoter (Fig. 4a). We observed that the full-length HSLUNN1 protein showed the highest efficiency in activating the TH promoter region compared to the two other variants (Fig. 4b). Notably, proteins lacking SUMO or LFn-ubiquitin, respectively (H-LUNN1 and HS-NN1), were each still able to activate the $\mathrm{TH}$ promoter, although to a much lower efficiency as compared to HS-LUNN1 full-length protein. The H-N1 protein (Nurr1 only) showed some basal but not significant levels of luciferase activation compared to the controls.

\section{Discussion}

In this study, we report the bacterial expression of human Nurr1 as a fusion protein composed of SUMO, ubiquitin as well as LFn for the cellular uptake. After exposure of HSLUNN1 protein to human dopaminergic SH-SY5Y cells, it became intracellularly cleaved by DUBs resulting in increased TH expression. TH promoter assay confirmed the transcriptional activity of the delivered Nurr1 protein. Furthermore, HS-LUNN1 protein strongly protected SH-SY5Y cells from 6-OHDA-induced cell death.

Current therapies for treating PD cannot restore dopaminergic neurons or stop their degeneration. Drug therapies are based on providing the dopamine precursor levodopa or on application of several inhibitors influencing the dopamine level [35]. As for surgical treatments, electrodes can be implanted for 


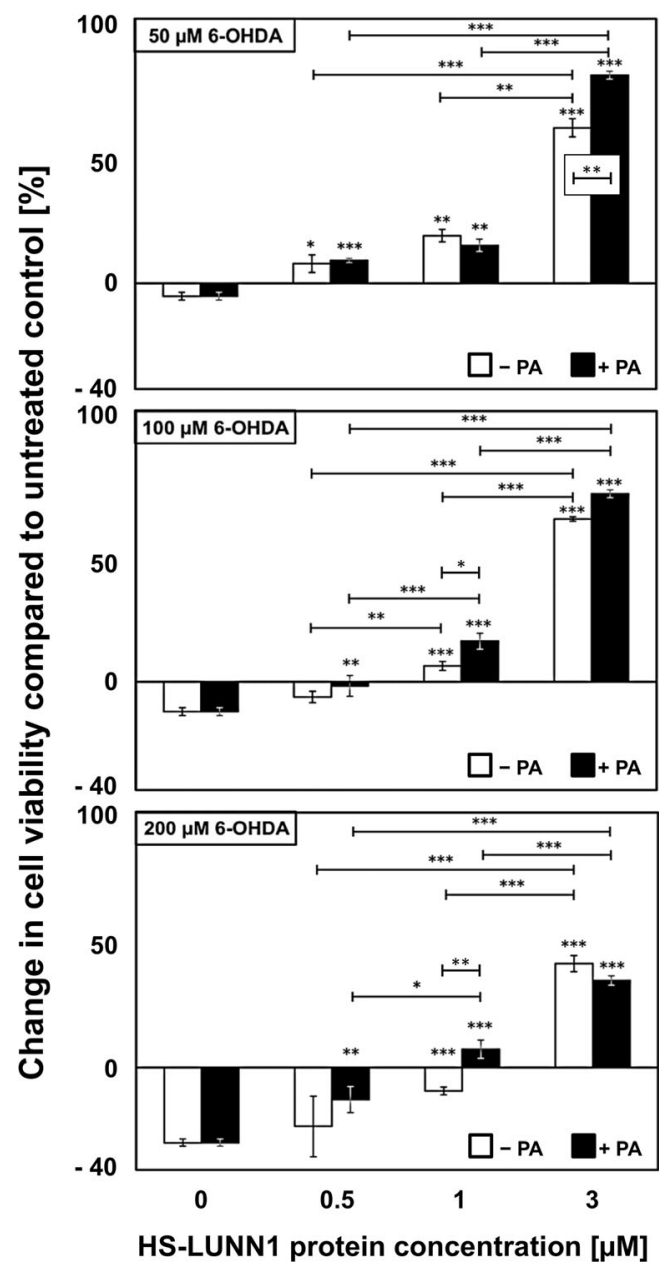

Fig. 3 Effects of HS-LUNN1 protein treatment ( \pm PA) on cell viability. SH-SY5Y cells were incubated for $24 \mathrm{~h}$ with HS-LUNN1 ( \pm PA) followed by treatment with 6-hydroxydopamine (6-OHDA) for $1 \mathrm{~h}$. Cell viability was quantified by RealTime-Glo ${ }^{\mathrm{TM}}$ MT cell viability assay (Promega). Luminescence signal of untreated cells (without protein and without toxin) was utilized as an internal standard and defined as zero to highlight deviations. Data are from six independent experiments, each of which was conducted in four replicates. Error bars indicate mean \pm SEM and asterisks above bars represent $p$ value compared to $0-\mu \mathrm{M}$ protein $\left({ }^{*} p<0.05 ; * * p<0.01 ; * * * p<0.001 ; n=6, t\right.$ test)

deep brain stimulation into the subthalamic nuclei, thalamus, or globus pallidus interna, thereby reducing the motor symptoms $[36,37]$, yet side effects cannot be excluded. Dopaminergic cell transplantation offers a future therapeutic option regarding PD, which is currently under investigation in animal models [38]. In this context, Nurr1 is used in combination with at least one additional transcription factor for restoring DA phenotypes in induced neurons [39, 40]. Caiazzo et al. showed that mouse and human fibroblast can be reprogrammed to functional induced dopaminergic neurons by applying the minimal set of the three transcription factors Mash1, Lmx1a, and Nurr1 [41]. More recently, overexpression of Nurr1 and Foxa2 transcription factors were shown to yield mature midbrain dopamine neurons from induced neural precursor cells [42].
The mentioned approaches are important steps towards the development of patient-derived induced dopaminergic neurons in cell replacement therapy for treating PD. However, this concept cannot be converted into a therapy as yet, because viral vectors (such as lentiviruses) carry an inherent risk for off target effects and immunogenicity [43]. A more promising approach could arise from the application of transcription factors via cellular delivery of the protein. As previously shown by Nagel et al., mesencephalic DA neurons could be protected from degeneration in several models of PD using transactivator of transcription (TAT)-mediated protein transduction of heat-shock protein 70 (TAT-Hsp70) [44]. Furthermore, reprogramming of human fibroblasts into induced dopaminergic neurons has been achieved by applying TAT-mediated Sox 2 and Lmx1a along with small molecules [45]. In addition, proteins fused to cell-penetrating peptide (CPP) or protein transduction domains (PTDs) [46] or to bacterial toxins [47] were developed. As application of bacterial toxins, mainly three different systems were used for cellular protein delivery, namely, diphtheria toxin, anthrax toxin, and Pseudomonas exotoxin. Several toxic proteins fused to anthrax-derived LFn were investigated as therapeutic tools for killing tumor cells [31, 48, 49]. Here, we decided to use LFn for the cellular delivery, supported by the observation that proteins fused to a bacterial toxin show a higher cytosolic delivery than CPPs [50].

In our study, Western blot analysis of total protein extractions from HS-LUNN1-treated SH-SY5Y cells showed cellular uptake and intracellular cleavage of HS-LUNN1 (Fig. 1bd). Thus, we observed an increase of delivered Nurrl fusion protein corresponding to the increasing concentrations of extracellular applied HS-LUNN1. This concentration-dependent increase in Nurrl was associated with a corresponding 4.8fold decline of endogenous Nur77 levels which is in partial agreement of data from Eelles et al. 2012, describing that haloperidol-induced upregulation of Nur77 and Nor1 was coupled to a reduction of Nurr1 [51]. Treatment with 6OHDA in SH-SY5Y cells leads to the upregulation, phosphorylation, and translocation of Nur77 form the nucleus to the mitochondria [52]. In contrast, knockdown of Nur77 reduces 6-OHDA-induced cell death, at least in PC12 cells [53], confirming a contra-directional coupling between Nur77 and Nurr1 [4]. A number of publications have shown the protective mechanisms of Nurr1 achieved by regulating mitochondrial genes such as superoxide dismutase 1 (SOD1), mitochondrial translation elongation factor, or cyclooxygenase $5 \beta$ $[4,54]$. The possible role played by the decreased proapoptotic Nur77 activity shown here (Fig. 1b) needs still to be investigated.

We observe here that exposure to HS-LUNN1 led to an increase in TH protein levels (Fig. 1d), which is a welldescribed downstream target of Nurr1 transcription factor [5]. In order to test if elevated $\mathrm{TH}$ levels resulted from 
a
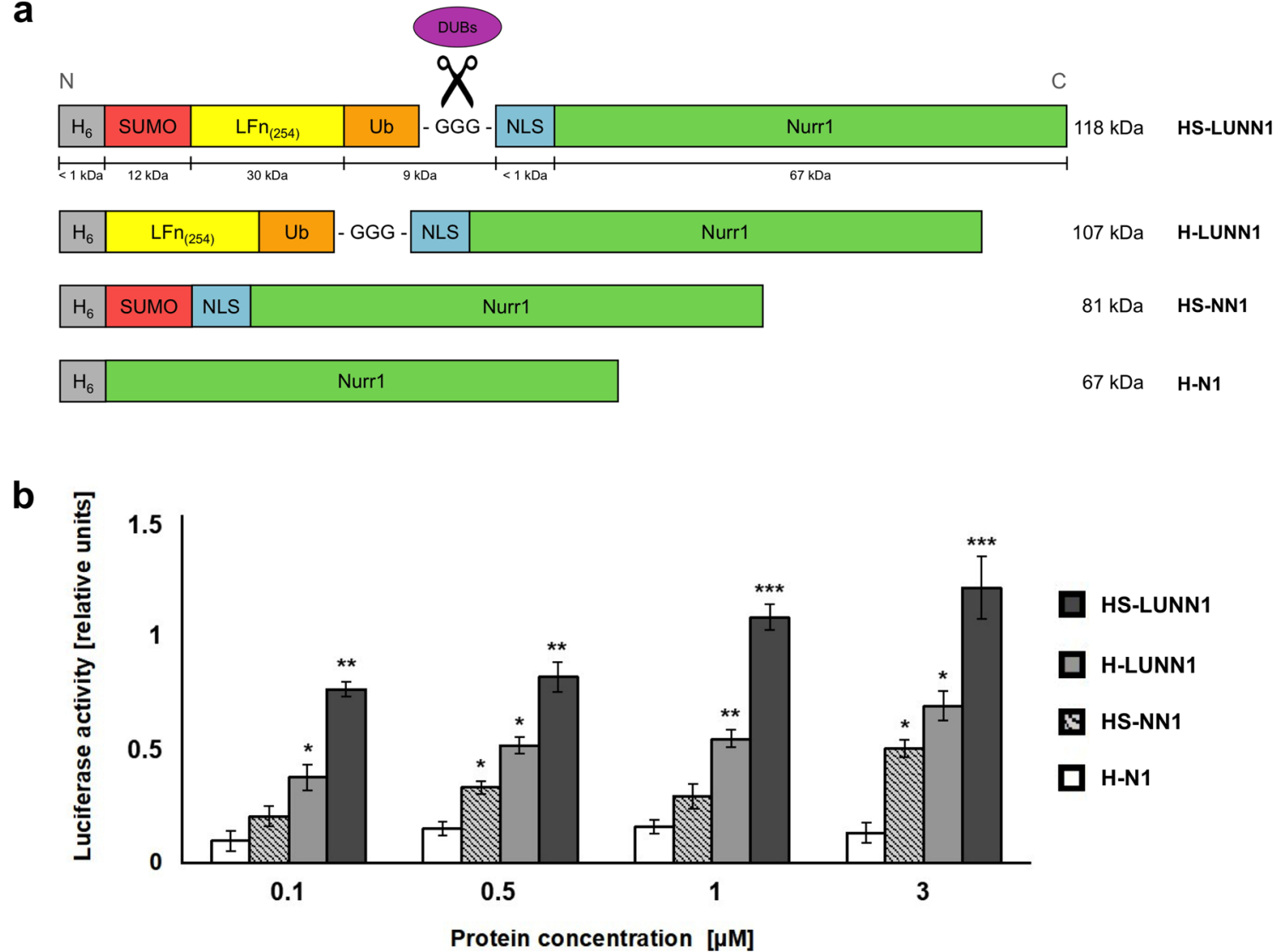

Fig. 4 Luciferase reporter assay in SH-SY5Y cells treated with HS-LUNN1 protein and its variants. a Domain structures of Nurr1 fusion protein variants. In addition to full-length HS-LUNN1 (Fig. 1a), variant Nurr1 fusion proteins were created as follows: without the SUMO domain (H-LUNN1), without the $\mathrm{LFn}_{(254)}$ and ubiquitin domain (HS-NN1), and with the hexahistidine tag fused to Nurrl only (H-N1). b Luciferase reporter assay in SH-SY5Y cells treated with various types of Nurrl fusion protein HS-LUNN1 as indicated

enhanced promoter activity, we used our previously described luciferase reporter assay of murine and human TH promoters [33]. The three tested concentrations of HS-LUNN1 showed similar activation rates for both promoters, but the human promoter showed a threefold stronger activation than the mouse promoter (Fig. 2).

As a working hypothesis, we propose that there is a cytosolic delivery of HS-LUNN1 (i) by cellular uptake through the plasma membrane, (ii) by release or escape from endosomes, or (iii) by direct LFn-mediated transmembrane delivery mechanism allowing Nurr1 to activate the TH promoter in the cell nucleus [55].

In accordance with other studies showing that the overexpression of Nurr1 leads to neuroprotection $[10,56]$, we asked whether HS-LUNN1 can exert similar cellular effects. Therefore, we treated SH-SY5Y cells with different concentrations of HS-LUNN1 in the presence or absence of PA and applied a neurotoxic stimulus by 6-OHDA (Fig. 3). While toxic 6-OHDA concentration showed the expected decrease in the figure. Cells were transfected with pTHh-pGl3B $24 \mathrm{~h}$ prior to protein delivery. The Renilla luciferase expression plasmid, pRL-TK, and the original pGL3B control were utilized as an internal and external standard, respectively. Data are obtained from three independent experiments, each of which was conducted in triplicate, and are means \pm SEM for untreated cells transfected with hTHp-pGl3B $\left(* p<0.05 ; *^{*} p<0.01 ; * * * p<0.001 ; n=3, t\right.$ test)

in cell viability of SH-SY5Y cells, increasing concentrations of HS-LUNN1 could counteract this toxic effect and could even increase cell viability of untreated cells. This clearly underlines that exogenous applied HS-LUNN1 fusion protein is capable to promote neuroprotection.

In a previous study, we demonstrated that transgenic activation of Ras activity in neurons led to increased Nurr1expression resulting in survival and differentiation of neurospheres into a dopaminergic cell fate [33]. All this underlines the important role of Nurr1 in enhancing and stabilizing DA properties in perspective for the development of cell replacement therapies.

While in general the application of PA is needed for LFnmediated cellular uptake, we found only minor effects when PA together with HS-LUNN1 was applied prior to the 6OHDA treatment (Fig. 3). All other experiments on HSLUNN1 delivery in this study were carried out in absence of PA. Such a PA-independent delivery is however in line to studies demonstrating that PA is not necessarily required for 
LFn-mediated cellular delivery of LFn fusion proteins when these are administered at concentrations $>1 \mu \mathrm{M}$ [23-25]. Accordingly, Cao et al. suggested that the cellular uptake of LFn fusion proteins can take place in absence of PA via another mechanism involving the major histocompatibility complex (MHC) class I pathway [22]. Here, the minor effect of PA might be explained that PA-dependent and independent cellular uptake mechanisms may occur in parallel. PA-dependent uptake may be more evident when PA and LFn are present at low concentrations corresponding to the receptor affinity of approximately $1 \mathrm{nM}[57,58]$.

Finally, we analyzed N-terminally His-tagged but truncated bacterially expressed variants of full-length HS-LUNN1. HLUNN1 (without SUMO domain) or HS-NN1 (without LFn and without ubiquitin) and H-N1 containing the Nurr1 protein without NLS were analyzed for their capacity of activating TH promoter (Fig. 4a). Again, the TH promoter-activity assay in SH-SY5Y cells was used to address the question of the differential contribution of the various domains to functional nuclear delivery. At all concentrations of the protein fragments tested, HS-LUNN1 showed the highest activation rate, meaning that all domains of the fusion protein were contributing to enhance transcriptional activity. Interestingly, H-N1 alone (containing His-tagged Nurrl only) was not sufficient to induce a concentration-dependent increase in luciferase activity suggesting that an endosomal/lysosomal mechanism of delivering extracellular His-tagged Nurr1 to the cytoplasm via leaky endosomal membrane compartments is unlikely [59]. Nurr1 contains an endogenous bipartite nuclear localization sequence [60], indicating that it has the capacity to enter the nucleus without additional NLS once delivered to the cytosol. Omitting the SUMO domain reduced the transcriptional activity by up to $50 \%$ at various concentrations of HS-LUNN1, suggesting that SUMO contributes to the efficiency of functional nuclear delivery. This is also supported by data obtained from the HS-NN1 protein showing that SUMO alone results in some functional delivery of NLS-Nurr1 without LFn domain. We cannot exclude here that the external N-terminal NLS plays a role in cellular delivery nor do we know if the possible effect of SUMO is due to its previously described ion channel activation or due to its capacity to enhance receptormediated internalization, nuclear localization, or any other effect [61]. Altogether, these results suggest that all the domains used contribute to the efficiency of functional nuclear transfer of Nurr1 transcription factor.

Taken together, human Nurr1 was fused to SUMO, ubiquitin, and the non-toxic $\mathrm{N}$ terminus of LFn for functional nuclear delivery, which was shown in the human dopaminergic cell line SH-SY5Y. TH promoter assays confirmed the transcriptional activity of full-length Nurrl fusion protein HS-LUNN1. Finally, applying HS-LUNN1 to human SHSY5Y cells led to a protection from neurotoxin 6-OHDAinduced cellular degeneration. These findings may have relevance for the nuclear delivery of Nurr1 transcription factor in the context of protein-based treatments in Parkinson's disease.

Acknowledgements We thank Victoria Trinkaus for experimental support.

Funding information We thank the European Union's Horizon 2020 research and innovation program under grant agreement No. 686841 (MAGNEURON) for funding to D.P., F.R., and R.H. This research was supported in part by the Intramural Program of the National Institute of Allergy and Infectious Diseases, NIH.

Open Access This article is distributed under the terms of the Creative Commons Attribution 4.0 International License (http:// creativecommons.org/licenses/by/4.0/), which permits unrestricted use, distribution, and reproduction in any medium, provided you give appropriate credit to the original author(s) and the source, provide a link to the Creative Commons license, and indicate if changes were made.

\section{References}

1. Giguere V (1999) Orphan nuclear receptors: from gene to function. Endocr Rev 20(5):689-725. https://doi.org/10.1210/edrv.20.5.0378

2. Liu J, Wang GH, Duan YH, Dai Y, Bao Y, Hu M, Zhou YQ, Li M et al (2017) Modulation of the Nur77-Bcl-2 apoptotic pathway by p38alpha MAPK. Oncotarget 8(41):69731-69745. https://doi.org/ 10.18632/oncotarget.19227

3. Yi M, Yang J, Li W, Li X, Xiong W, McCarthy JB, Li G, Xiang B (2017) The NOR1/OSCP1 proteins in cancer: from epigenetic silencing to functional characterization of a novel tumor suppressor. $\mathrm{J}$ Cancer 8(4):626-635. https://doi.org/10.7150/jca.17579

4. Wei XB, Gao HM, Zou J, Liu X, Chen D, Liao JC, Xu YQ, Ma L et al (2016) Contra-directional coupling of Nur77 and Nurr1 in neurodegeneration: a novel mechanism for memantine-induced antiinflammation and anti-mitochondrial impairment. Mol Neurobiol 53(9):5876-5892. https://doi.org/10.1007/s12035-015-9477-7

5. Jankovic J, Chen S, Le WD (2005) The role of Nurr1 in the development of dopaminergic neurons and Parkinson's disease. Prog Neurobiol 77(1-2):128-138. https://doi.org/10.1016/j.pneurobio. 2005.09.001

6. Saucedo-Cardenas O, Quintana-Hau JD, Le WD, Smidt MP, Cox JJ, DeMayo F, Burbach JPH, Conneely OM (1998) Nurrl is essential for the induction of the dopaminergic phenotype and the survival of ventral mesencephalic late dopaminergic precursor neurons. Proc Natl Acad Sci U S A 95(7):4013-4018. https://doi.org/ 10.1073/pnas.95.7.4013

7. Castelo-Branco GA, Wagner J, Rodriguez FJ, Kele J, Sousa K, Rawal N, Pasolli HA, Fuchs E et al (2003) Differential regulation of midbrain dopaminergic neuron development by Wnt-1, Wnt-3a, and Wnt-5a. Proc Natl Acad Sci U S A 100(22):12747-12752. https://doi.org/10.1073/pnas.1534900100

8. Klein C, Westenberger A (2012) Genetics of Parkinson's disease. Cold Spring Harb Perspect Med 2(1):a008888. https://doi.org/10. 1101/cshperspect.a008888

9. Chu Y, Kompoliti K, Cochran EJ, Mufson EJ, Kordower JH (2002) Age-related decreases in Nurr1 immunoreactivity in the human substantia nigra. J Comp Neurol 450(3):203-214. https://doi.org/ 10.1002/cne.10261

10. Liu W, Gao Y, Chang N (2017) Nurr1 overexpression exerts neuroprotective and anti-inflammatory roles via down-regulating CCL2 expression in both in vivo and in vitro Parkinson's disease 
models. Biochem Biophys Res Commun 482(4):1312-1319. https://doi.org/10.1016/j.bbrc.2016.12.034

11. Dong J, Li S, Mo JL, Cai HB, Le WD (2016) Nurr1-based therapies for Parkinson's disease. CNS Neurosci Ther 22(5):351-359. https:// doi.org/10.1111/cns.12536

12. Oh SM, Chang MY, Song JJ, Rhee YH, Joe EH, Lee HS, Yi SH, Lee SH (2015) Combined Nurr1 and Foxa2 roles in the therapy of Parkinson's disease. EMBO Mol Med 7(5):510-525. https://doi. org/10.15252/emmm.201404610

13. Wang J, Bi W, Zhao W, Varghese M, Koch RJ, Walker RH, Chandraratna RA, Sanders ME et al (2016) Selective brain penetrable Nurr1 transactivator for treating Parkinson's disease. Oncotarget 7(7):7469-7479. https://doi.org/10.18632/oncotarget. 7191

14. Leppla SH (2013) Chapter 281 - Anthrax lethal factor A2 Rawlings, Neil D. In: Salvesen G (ed) Handbook of proteolytic enzymes. Academic Press, pp 1257-1261. https://doi.org/10. 1016/B978-0-12-382219-2.00282-9

15. Liu SH, Zhang Y, Hoover B, Leppla SH (2013) The receptors that mediate the direct lethality of anthrax toxin. Toxins 5(1):1-8. https://doi.org/10.3390/toxins5010001

16. Kintzer AF, Thoren KL, Sterling HJ, Dong KC, Feld GK, Tang II, Zhang TT, Williams ER et al (2009) The protective antigen component of anthrax toxin forms functional octameric complexes. J Mol Biol 392(3):614-629. https://doi.org/10.1016/j.jmb.2009.07. 037

17. Zhao J, Milne JC, Collier RJ (1995) Effect of anthrax toxin's lethal factor on ion channels formed by the protective antigen. J Biol Chem 270(31):18626-18630. https://doi.org/10.1074/jbc.270.31. 18626

18. Jiang J, Pentelute BL, Collier RJ, Zhou ZH (2015) Atomic structure of anthrax protective antigen pore elucidates toxin translocation. Nature 521(7553):545-549. https://doi.org/10.1038/nature14247

19. Tamayo AG, Slater L, Taylor-Parker J, Bharti A, Harrison R, Hung DT, Murphy JR (2011) GRP78(BiP) facilitates the cytosolic delivery of anthrax lethal factor (LF) in vivo and functions as an unfoldase in vitro. Mol Microbiol 81(5):1390-1401. https://doi. org/10.1111/j.1365-2958.2011.07770.x

20. Arora N, Klimpel KR, Singh Y, Leppla SH (1992) Fusions of anthrax toxin lethal factor to the ADP-ribosylation domain of Pseudomonas exotoxin-a are potent cytotoxins which are translocated to the cytosol of mammalian-cells. J Biol Chem 267(22):15542-15548

21. Arora N, Leppla SH (1993) Residues 1-254 of anthrax toxin lethal factor are sufficient to cause cellular uptake of fused polypeptides. J Biol Chem 268(5):3334-3341

22. Cao H, Agrawal D, Kushner N, Touzjian N, Essex M, Lu Y (2002) Delivery of exogenous protein antigens to major histocompatibility complex class I pathway in cytosol. J Infect Dis 185(2):244-251. https://doi.org/10.1086/338448

23. Kushner N, Zhang D, Touzjian N, Essex M, Lieberman J, Lu YC (2003) A fragment of anthrax lethal factor delivers proteins to the cytosol without requiring protective antigen. Proc Natl Acad Sci U S A 100(11):6652-6657. https://doi.org/10.1073/pnas.1131930100

24. McEvers K, Elrefaei M, Norris P, Deeks S, Martin J, Lu Y, Cao H (2005) Modified anthrax fusion proteins deliver HIV antigens through MHC class I and II pathways. Vaccine 23(32):41284135. https://doi.org/10.1016/j.vaccine.2005.03.022

25. Shaw CA, Starnbach MN (2008) Both CD4(+) and CD8(+) T cells respond to antigens fused to anthrax lethal toxin. Infect Immun 76(6):2603-2611. https://doi.org/10.1128/Iai.01718-07

26. Pichler A, Fatouros C, Lee H, Eisenhardt N (2017) SUMO conjugation - a mechanistic view. Biomol Concepts 8(1):13-36. https:// doi.org/10.1515/bmc-2016-0030

27. Marblestone JG, Edavettal SC, Lim Y, Lim P, Zuo X, Butt TR (2006) Comparison of SUMO fusion technology with traditional gene fusion systems: enhanced expression and solubility with SUMO. Protein Sci 15(1):182-189. https://doi.org/10.1110/ps. 051812706

28. Varshavsky A (2005) Ubiquitin fusion technique and related methods. Methods Enzymol 399:777-799. https://doi.org/10. 1016/S0076-6879(05)99051-4

29. Brzovic PS, Lissounov A, Christensen DE, Hoyt DW, Klevit RE (2006) A UbcH5/ubiquitin noncovalent complex is required for processive BRCA1-directed ubiquitination. Mol Cell 21(6):873880. https://doi.org/10.1016/j.molcel.2006.02.008

30. Rueden CT, Schindelin J, Hiner MC, DeZonia BE, Walter AE, Arena ET, Eliceiri KW (2017) ImageJ2: ImageJ for the next generation of scientific image data. BMC Bioinformatics 18(1):529. https://doi.org/10.1186/s12859-017-1934-z

31. Bachran C, Morley T, Abdelazim S, Fattah RJ, Liu S, Leppla SH (2013) Anthrax toxin-mediated delivery of the Pseudomonas exotoxin A enzymatic domain to the cytosol of tumor cells via cleavable ubiquitin fusions. MBio 4(3):e00201-e00213. https://doi.org/ 10.1128/mBio.00201-13

32. Johnson ES (2004) Protein modification by SUMO. Annu Rev Biochem 73:355-382. https://doi.org/10.1146/annurev.biochem. 73.011303.074118

33. Chakrabarty K, Serchov T, Mann SA, Dietzel ID, Heumann R (2007) Enhancement of dopaminergic properties and protection mediated by neuronal activation of Ras in mouse ventral mesencephalic neurones. Eur J Neurosci 25(7):1971-1981. https://doi.org/ 10.1111/j.1460-9568.2007.05457.x

34. Michel PP, Hefti F (1990) Toxicity of 6-hydroxydopamine and dopamine for dopaminergic neurons in culture. J Neurosci Res 26(4):428-435. https://doi.org/10.1002/jnr.490260405

35. Oertel WH (2017) Recent advances in treating Parkinson's disease. F1000Res 6:260. https://doi.org/10.12688/f1000research.10100.1

36. Bronstein JM, Tagliati M, Alterman RL, Lozano AM, Volkmann J, Stefani A, Horak FB, Okun MS et al (2011) Deep brain stimulation for Parkinson disease: an expert consensus and review of key issues. Arch Neurol 68(2):165. https://doi.org/10.1001/archneurol. 2010.260

37. Heumann R, Moratalla R, Herrero MT, Chakrabarty K, DruckerColin R, Garcia-Montes JR, Simola N, Morelli M (2014) Dyskinesia in Parkinson's disease: mechanisms and current nonpharmacological interventions. J Neurochem 130(4):472-489. https://doi.org/10.1111/jnc.12751

38. Muller J, Ossig C, Greiner JF, Hauser S, Fauser M, Widera D, Kaltschmidt C, Storch A et al (2015) Intrastriatal transplantation of adult human neural crest-derived stem cells improves functional outcome in parkinsonian rats. Stem Cells Transl Med 4(1):31-43. https://doi.org/10.5966/sctm.2014-0078

39. Tan X, Zhang L, Qin J, Tian M, Zhu H, Dong C, Zhao H, Jin G (2013) Transplantation of neural stem cells co-transfected with Nurr1 and Brn4 for treatment of parkinsonian rats. Int J Dev Neurosci 31(1):82-87. https://doi.org/10.1016/j.ijdevneu.2012.10. 007

40. Ko TL, Fu YY, Shih YH, Lin YH, Ko MH, Fu TW, Lin TY, Hsiao HS et al (2015) A high-efficiency induction of dopaminergic cells from human umbilical mesenchymal stem cells for the treatment of hemiparkinsonian rats. Cell Transplant 24(11):2251-2262. https:// doi.org/10.3727/096368914X685078

41. Caiazzo M, Dell'Anno MT, Dvoretskova E, Lazarevic D, Taverna S, Leo D, Sotnikova TD, Menegon A et al (2011) Direct generation of functional dopaminergic neurons from mouse and human fibroblasts. Nature 476(7359):224-227. https://doi.org/10.1038/ nature 10284

42. Lim MS, Chang MY, Kim SM, Yi SH, Suh-Kim H, Jung SJ, Kim MJ, Kim JH et al (2015) Generation of dopamine neurons from rodent fibroblasts through the expandable neural precursor cell 
stage. J Biol Chem 290(28):17401-17414. https://doi.org/10.1074/ jbc.M114.629808

43. Thomas CE, Ehrhardt A, Kay MA (2003) Progress and problems with the use of viral vectors for gene therapy. Nat Rev Genet 4(5): 346-358. https://doi.org/10.1038/nrg1066

44. Nagel F, Falkenburger BH, Tonges L, Kowsky S, Poppelmeyer C, Schulz JB, Bahr M, Dietz GP (2008) Tat-Hsp70 protects dopaminergic neurons in midbrain cultures and in the substantia nigra in models of Parkinson's disease. J Neurochem 105(3):853-864. https://doi.org/10.1111/j.1471-4159.2007.05204.x

45. Mirakhori F, Zeynali B, Rassouli H, Salekdeh GH, Baharvand H (2015) Direct conversion of human fibroblasts into dopaminergic neural progenitor-like cells using TAT-mediated protein transduction of recombinant factors. Biochem Biophys Res Commun 459(4):655-661. https://doi.org/10.1016/j.bbrc.2015.02.166

46. Bolhassani A, Jafarzade BS, Mardani G (2017) In vitro and in vivo delivery of therapeutic proteins using cell penetrating peptides. Peptides 87:50-63. https://doi.org/10.1016/j.peptides.2016.11.011

47. Beilhartz GL, Sugiman-Marangos SN, Melnyk RA (2017) Repurposing bacterial toxins for intracellular delivery of therapeutic proteins. Biochem Pharmacol 142:13-20. https://doi.org/10. 1016/j.bcp.2017.04.009

48. Martin EW, Buzza MS, Driesbaugh KH, Liu S, Fortenberry YM, Leppla SH, Antalis TM (2015) Targeting the membrane-anchored serine protease testisin with a novel engineered anthrax toxin prodrug to kill tumor cells and reduce tumor burden. Oncotarget 6(32):33534-33553. https://doi.org/10.18632/oncotarget.5214

49. Bachran C, Leppla SH (2016) Tumor targeting and drug delivery by anthrax toxin. Toxins (Basel) 8(7). https://doi.org/10.3390/ toxins 8070197

50. Verdurmen WPR, Mazlami M, Pluckthun A (2017) A quantitative comparison of cytosolic delivery via different protein uptake systems. Sci Rep 7(1):13194. https://doi.org/10.1038/s41598-01713469-y

51. Eells JB, Wilcots J, Sisk S, Guo-Ross SX (2012) NR4A gene expression is dynamically regulated in the ventral tegmental area dopamine neurons and is related to expression of dopamine neurotransmission genes. J Mol Neurosci 46(3):545-553. https://doi.org/ 10.1007/s12031-011-9642-Z

52. No H, Bang Y, Lim J, Kim SS, Choi HS, Choi HJ (2010) Involvement of induction and mitochondrial targeting of orphan nuclear receptor Nur77 in 6-OHDA-induced SH-SY5Y cell death.
Neurochem Int 56(4):620-626. https://doi.org/10.1016/j.neuint. 2010.01.005

53. Gao HM, Chen ZY, Fu YM, Yang XY, Weng RH, Wang R, Lu JJ, Pan MQ et al (2016) Nur77 exacerbates PC12 cellular injury in vitro by aggravating mitochondrial impairment and endoplasmic reticulum stress. Sci Rep Uk 6:ARTN 34403. https://doi.org/10. 1038/srep34403

54. Decressac M, Volakakis N, Bjorklund A, Perlmann T (2013) NURR1 in Parkinson disease-from pathogenesis to therapeutic potential. Nat Rev Neurol 9(11):629-636. https://doi.org/10.1038/ nrneurol.2013.209

55. Kim KS, Kim CH, Hwang DY, Seo H, Chung S, Hong SJ, Lim JK, Anderson $T$ et al (2003) Orphan nuclear receptor Nurrl directly transactivates the promoter activity of the tyrosine hydroxylase gene in a cell-specific manner. J Neurochem 85(3):622-634

56. Li QJ, Tang YM, Liu J, Zhou DY, Li XP, Xiao SH, Jian DX, Xing YG (2007) Treatment of Parkinson disease with C17.2 neural stem cells overexpressing NURR1 with a recombined republic-deficit adenovirus containing the NURR1 gene. Synapse 61(12):971977. https://doi.org/10.1002/syn.20449

57. Ballard JD, Collier RJ, Starnbach MN (1996) Anthrax toxinmediated delivery of a cytotoxic T-cell epitope in vivo. Proc Natl Acad Sci U S A 93(22):12531-12534. https://doi.org/10.1073/ pnas.93.22.12531

58. Ballard JD, Doling AM, Beauregard K, Collier RJ, Starnbach MN (1998) Anthrax toxin-mediated delivery in vivo and in vitro of a cytotoxic T-lymphocyte epitope from ovalbumin. Infect Immun 66(2):615-619

59. D'Astolfo DS, Pagliero RJ, Pras A, Karthaus WR, Clevers H, Prasad V, Lebbink RJ, Rehmann H et al (2015) Efficient intracellular delivery of native proteins. Cell 161(3):674-690. https://doi. org/10.1016/j.cell.2015.03.028

60. Garcia-Yague AJ, Rada P, Rojo AI, Lastres-Becker I, Cuadrado A (2013) Nuclear import and export signals control the subcellular localization of Nurr1 protein in response to oxidative stress. J Biol Chem 288(8):5506-5517. https://doi.org/10.1074/jbc.M112. 439190

61. Creton S, Jentsch S (2010) Snapshot: the SUMO system. Cell 143(5):848- e841. https://doi.org/10.1016/j.cell.2010.11.026

62. Catic A, Misaghi S, Korbel GA, Ploegh HL (2007) ElaD, a deubiquitinating protease expressed by E. coli. PLoS One 2(4): e381. https://doi.org/10.1371/journal.pone.0000381 